\title{
Length scale dependence in elastomers - comparison of indentation experiments with numerical simulations
}

\author{
Nitin Garg, Chung-Souk Han, ${ }^{*}$ and Farid Alisafaei ${ }^{\dagger}$ \\ Department of Mechanical Engineering, University of Wyoming, Laramie, WY 82071, USA
}

\begin{abstract}
Probing depth dependent deformation at nano- and micrometer length scales has been observed in indentation experiments of polymers. Unlike in metals, where size effects are observed in plastic deformation and are attributed to geometrically necessary dislocations, the origin of size dependence in polymers is not well understood. As classical continuum theories are unable to describe such phenomena, higher order gradient theories have been developed to capture such size dependent deformation behavior. The present study adopts the penalty finite element approach for a couple stress elasticity theory under axisymmetric conditions to numerically simulate and analyze the probing depth dependent deformation. Polydimethylsiloxane (PDMS) and natural rubber have been used as model materials to analyze the depth dependent deformation at different probing depths. Simulations were performed on PDMS using spherical indenter tips of different radii to show the influence of strain/rotation gradients on elastic modulus. To capture the experimentally observed increase in hardness with decreasing probing depth, simulations applying a conical indenter tip were performed and compared with experimental data.
\end{abstract}

Keywords: polymers, nanoindentation, length scale dependent deformation, finite elements

\section{Introduction}

Depth sensing indentation testing has been widely applied to determine hardness, elastic modulus, as well as to study deformation mechanisms at micro- and nanometer length scales. Numerous indentation experiments conducted on metals [1-3] and polymers [4-16] demonstrated that the hardness is significantly higher at small probing depths. In $[11,13,17]$ the length scale dependence in polymers has been experimentally observed in elastic deformation, which is in contrast to metals, where length scale effects were observed in plastic deformation and usually attributed to geometrically necessary dislocations [2]. Although there is mounting experimental evidence for size effects in polymers [4-20],

*Corresponding author, corresponding address: C.-S. Han, Dept. 3295, University of Wyoming, Laramie, WY 82071, USA. Email: chan1@uwyo.edu

${ }^{\dagger}$ Current address: F. Alisafaei, Department of Material Science and Engineering, University of Pennsylvania, Philadelphia, PA 19104, USA. Email: alisafae@seas.upenn.edu 
compared to metals, length scale dependent deformation in polymers is arguably not well understood and there are only few length scale dependent theories suggested in the literature $[5,6,21,22]$ for polymers.

Length scale dependent phenomena cannot be explained nor predicted by classical, local continuum theories. This has led to the development of phenomenological continuum theories with higher order gradients in the displacements where the material length scales are introduced into the deformation energy [23-26]. Despite considerable theoretical and computational contributions on the analysis of length scale dependent phenomena with higher-order gradient theories, there are only very limited numerical studies $[27,28]$ in the literature that examine the probing depth dependent deformation in polymers.

It has also been observed that the determined elastic moduli of some polymers like epoxy [12], polydimethylsiloxane (PDMS) [13] and natural rubber [14] increases with decreasing depth when Sneddon's theory together with a pyramidal tip is applied, whereas no change in the determined elastic moduli of these polymers have been observed with depth when the Hertz theory along with a spherical tip was applied. It was hypothesized that the differences between the determined elastic moduli using spherical and pyramidal tips are attributed to the higher order gradients of displacements, as it is argued that these gradients remain essentially constant with a spherical tip, while applying a conical/pyramidal tip these gradients increase with decreasing probing depth.

The goal of this paper is to compare the experimental results with numerical simulations based on the axisymmetric couple stress approach developed in [29] and to examine whether the change in hardness with respect to the probing depth can be predicted by the couple stress elasticity theory. Simulations were carried out with spherical and conical indenter (equivalent to pyramidal tip) tips on PDMS as a model material and compared with the experiments to interpret the probing depth dependent deformation behavior by considering the rotation gradients. Also, the simulations with a conical tip were performed on natural rubber and compared with the experimental data. In the next section, results obtained from the indentation type experiments are briefly discussed. In the section thereafter, the influence of the rotation gradients with respect to different tip geometries is suggested and a rotation gradient model to predict such a size dependent behavior (when conical tips are applied) is discussed. The penalty approach of the applied couple stress axisymmetric elasticity theory of [29] is then applied to perform numerical simulations on PDMS and natural rubber at different probing depths using different tip geometries.

\section{Experiments}

The universal hardness $H_{\mathrm{U}}$, also known as Martens hardness, is obtained from load-displacement data and can be calculated in accordance with ISO 14577-1 [30] as follows

$$
H_{\mathrm{U}}=\frac{F_{\max }}{A_{\mathrm{s}}} \quad,
$$

where $A_{\mathrm{s}}$ is the nominal surface area of the indenter tip penetrating beyond initial contact with the material sample under the maximum applied force $F_{\max }$. For the Berkovich indenter tip, the nominal surface area is given as

$$
A_{\mathrm{s}}=C h^{2} \quad,
$$

where $h$ corresponds to the probing depth at $F_{\max }$ and $C$ is a constant equal to 26.43 . It should be noted that the expression "indentation" is usually applied to tests inducing permanent residual impressions on the sample. The experiments on elastomers in [11, 13, 14], however, showed almost pure reversible, elastic deformation after fully removing the load. Therefore, we adopt "probing depth" in this article to 
describe the experimental results and their corresponding numerical simulations. It can be seen in Fig. 1 that the ratio of the inelastic deformation work (area enclosed by the curve) to the total deformation work is small indicating the elastic nature of the deformation mechanism during nanoindentation of PDMS.

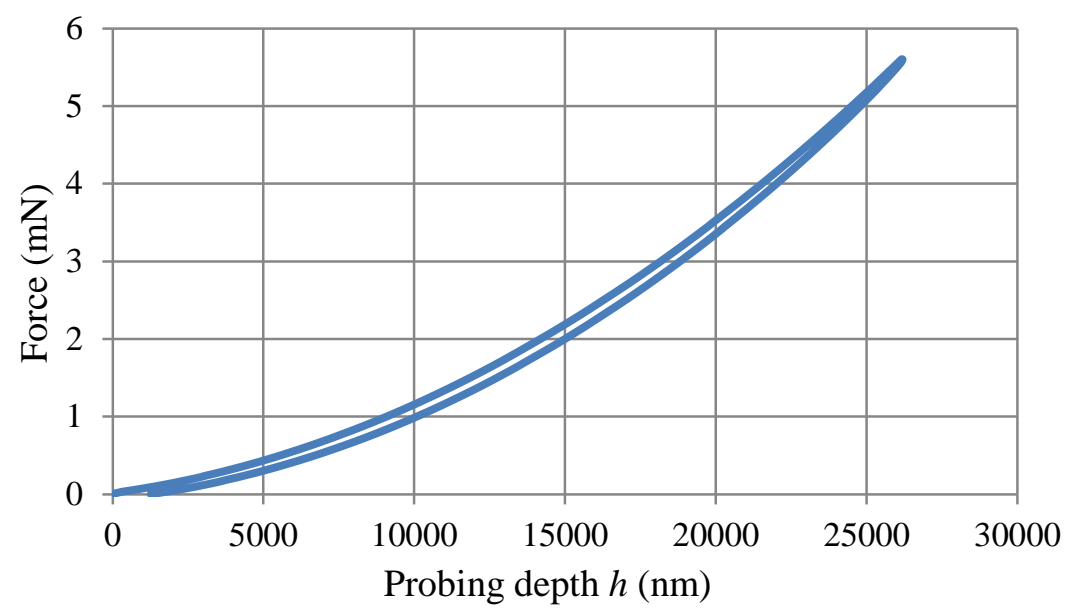

Fig. 1. Force versus probing depth $h$ with the Berkovich tip (unpublished data of experiments in [11]).

Unlike the indentation hardness [31], which is evaluated by a projection technique to approximate the contact area of the tip with the sample at the maximum depth, the universal hardness is defined as the force divided by the nominal surface area (the area that is nominally below the initial surface). In this respect, it should be noted that the indentation hardness has been originally developed for ceramics and metallic materials subjected to plastic/permanent deformation and may not be applicable for the highly elastic materials considered here $[8,30]$. As the universal hardness $H_{\mathrm{U}}$ incorporates both plastic and elastic deformations, it is actually applicable to all materials.

The indentation type experiments conducted on PDMS in [11] resulted in an increase in $H_{\mathrm{U}}$ (from 0.28 to $0.44 \mathrm{MPa}$ ) with decreasing $h$ (from 31.5 to $6.0 \mu \mathrm{m}$ ). Recent experiments [12-14] exploring size dependence associated with probing of polymers with a diamond spherical tip have revealed that for a fixed radius of a spherical indenter tip, the determined elastic moduli showed negligible changes with the changing probing depth, i.e. elastic modulus is independent of $h$. The elastic modulus $E_{\mathrm{P}}$ of PDMS in [11] was determined according to the Hertzian theory and Sneddon's theory by applying spherical and pyramidal Berkovich tips, respectively. It was assumed that both spherical (curvature radius of $250 \mu \mathrm{m}$ ) and Berkovich tips are rigid as the elastic modulus of tip material (diamond) is many orders of magnitude higher than that of PDMS.

Hardly any changes in determined elastic moduli of PDMS were observed experimentally when spherical indenter tips were applied at different probing depths. According to the Hertzian theory [32], the elastic modulus of the polymer $E_{\mathrm{p}}^{\mathrm{Hertz}}$ can be obtained as

$$
E_{\mathrm{p}}^{\text {Hertz }}=\left(1-v_{\mathrm{p}}^{2}\right) E_{\mathrm{r}},
$$

where $v_{\mathrm{p}}$ is the Poisson's ratio of the polymer and $E_{\mathrm{r}}$ is the reduced elastic modulus obtained from the load-displacement curve as

$$
E_{\mathrm{r}}=\frac{3}{4} \frac{F}{R^{2}}\left(\frac{R}{h}\right)^{\frac{3}{2}}
$$


where $F$ is the applied force, $R$ the curvature radius of the spherical tip, and assuming linear elasticity and a small ratio of contact area to $R$. In contrast to spherical tips, the elastic modulus determined with a conical tip applying Sneddon's theory [33] $E_{\mathrm{p}}^{\text {Sneddon }}$ was found to increase (from 2.64 to 4.68 MPa) with decreasing $h$ (from 31.5 to $6.0 \mu \mathrm{m}$ ). According to Sneddon's theory for frictionless indentation of elastic materials with conical tip, the elastic modulus can be determined from load-displacement relation as

$$
E_{\mathrm{p}}^{\text {Sneddon }}=\frac{F \pi\left(1-v_{\mathrm{p}}^{2}\right)}{2 \tan \alpha h^{2}},
$$

in which $\alpha=70.3^{\circ}$ is a half angle defining a cone equivalent to a Berkovich tip. For reference, the results obtained from these experiments are illustrated later.
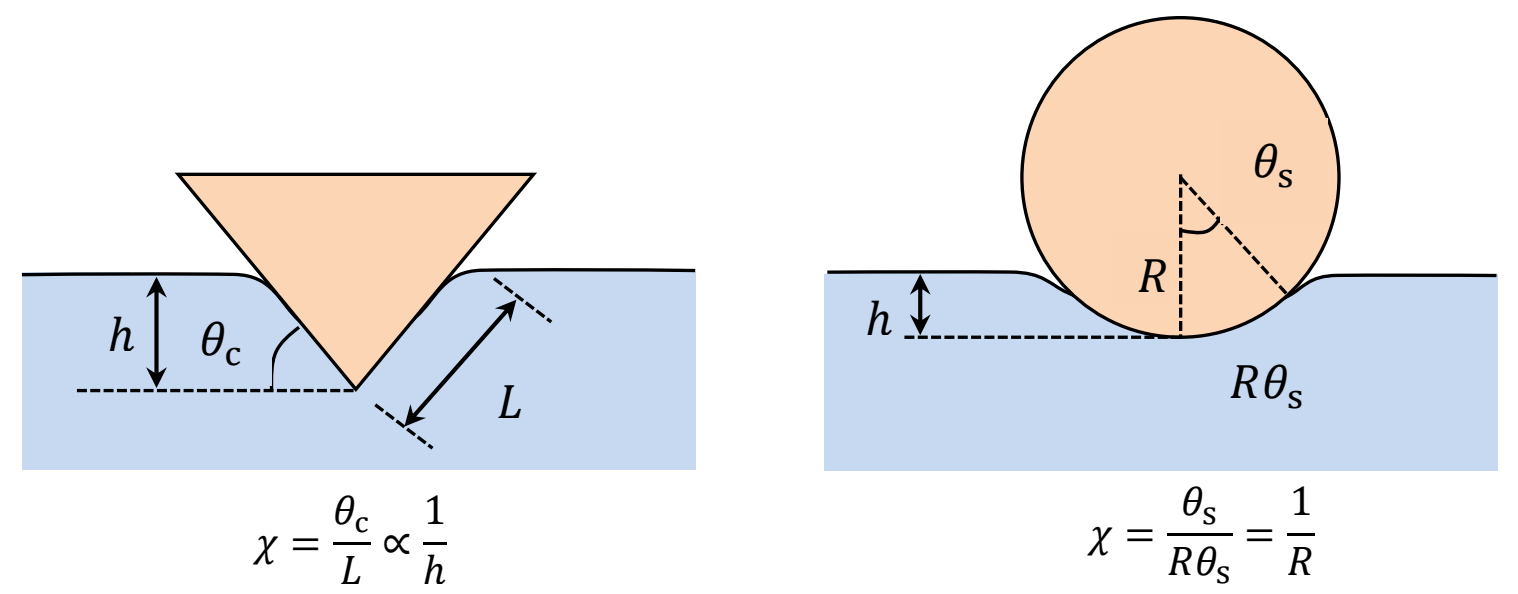

Fig. 2. Rotations and their gradients for conical and spherical tips and their dependence on $h$.

\section{Non-local, rotation gradient theory}

To predict the length scale effects mentioned above, atomistic simulations may be tempting, as length scale effects have been found to be dependent on polymer molecular structure [7, 15]. While molecular simulations may provide some basis for general trends [34, 35], they have their limitations as (i) deformation in polymers is highly dissipative and require large time scales, so that extreme computation times are necessary merely to determine atomistic positions prior to any load induced deformation and (ii) these effects occur at length scales of more than $1 \mu \mathrm{m}$. Therefore, atomistic simulations that can be quantitatively compared with experiments are arguably unrealistic for time being.

Applying geometrical analyses [2,36], indentation testing with indenters of self-similar geometries, like cones or pyramids, would result in an increase in higher order displacement gradients with decreasing $h$. In contrast, indentations with a spherical tip would cause negligible changes in the higher order displacement gradients with decreasing $h$. Fig. 2 illustrates the change in rotation gradients denoted as $\chi$ (as a form of higher order displacement gradients) with respect to $h$ for conical and spherical tips. As motivated therein, the rotation gradients should be approximately inversely proportional to $h$ applying a conical tip and therefore rotation gradients increase with $h$. For a spherical tip, the rotation gradients should be approximately inversely proportional to the tip radius $R$ and essentially independent of $h$.

To examine the influence of rotation gradients $\chi$ on the depth dependent deformation, comparative indentation studies have been performed with both Berkovich and spherical indenter tips in [10, 11]. The experiments therein were in agreement with the association of depth dependent deformation to rotation 
gradients $\chi$ as for conical/pyramidal tips measured hardness and elastic moduli increased with decreasing $h$, while such depth dependence was absent for spherical tips. From an energetic viewpoint, the total indentation work $W_{\mathrm{I}}$ can be divided into elastic work $W_{\mathrm{I}}^{\mathrm{e}}$ and inelastic work $W_{\mathrm{I}}^{\mathrm{ie}}$ as $W_{\mathrm{I}}=W_{\mathrm{I}}^{\mathrm{e}}+W_{\mathrm{I}}^{\mathrm{ie}}$. Due to negligible inelastic deformation observed experimentally in elastomers such as PDMS [11, 13], $W_{\mathrm{I}}^{\text {ie }}$ is neglected in the following yielding $W_{\mathrm{I}}=W_{\mathrm{I}}^{\mathrm{e}}$ for the total deformation work. In [21] the observed increase in the hardness of polymers was interpreted with rotation gradients in terms of a non-local component $\widehat{W}_{\mathrm{I}}^{\mathrm{F}}$ arising from the bending rigidity of the polymer chains and their interactions [15, 21, 22]. The total indentation work $W_{\mathrm{I}}$ can be then be decomposed as

$$
W_{\mathrm{I}}=W_{\mathrm{I} \varepsilon}^{\mathrm{e}}+\widehat{W}_{\mathrm{I}}^{\mathrm{F}},
$$

where $W_{\mathrm{I} \varepsilon}^{\mathrm{e}}$ is the local elastic work (the subscript $\varepsilon$ indicates the local, strain related nature of this component). Due to the reversible nature of the non-local term $\widehat{W}_{\mathrm{I}}^{\mathrm{F}}$, the total indentation work $W_{\mathrm{I}}$ is considered to be elastic, i.e. $W_{\mathrm{I}}^{\mathrm{e}}=W_{\mathrm{I}}$. According to [21, 22], due to increases in the rotation gradients at small depths $h$, an additional energy density $\widehat{W}^{\mathrm{F}}$ will be stored and consequently the additional work (applying Einstein's summation convention)

$$
\widehat{W}_{\mathrm{I}}^{\mathrm{F}}=\int_{V} \widehat{W}^{\mathrm{F}} d V=\int_{V} \frac{\widehat{K}}{3} \chi_{i j}^{\mathrm{s}} \chi_{i j}^{\mathrm{s}} d V
$$

should be exerted, where $\chi_{i j}^{\mathrm{s}}=\left(\chi_{i j}+\chi_{j i}\right) / 2$ is the symmetric part of the rotation gradient tensor defined as $\chi_{i j}=e_{i n m} u_{m, j n} / 2$ with displacement field $u_{m}$ and permutation symbol $e_{i n m}$, and $\widehat{K}$ is a length scale material parameter that can be micromechanically motivated $[21,22,34]$ and is believed to be dependent on the bending stiffness of the polymer chains, cross link density, and molecular weight. When compared to the couple stress theory of Yang et al. [37] the length scale parameter can be given as $\widehat{K}=3 \mu \ell^{2}$, with shear modulus $\mu$ and length scale parameter $\ell$. Therefore, the increases in $\widehat{W}^{\mathrm{F}}$ with decreasing $h$ for conical tips could predict the differences between $E_{\mathrm{p}}^{\text {Hertz }}$ and $E_{\mathrm{p}}^{\text {Sneddon }}$ in the experiments. As Sneddon's theory does not consider rotation gradient effects induced by a pyramidal tip, it would overestimate the actual elastic modulus due to the additional stiffness induced by the bending stiffness of the polymer chains and their interactions. For macroscopic $h$ and $R$, these gradients become negligible for pyramidal/conical and spherical tips, respectively, so that Sneddon's ( $\left.E_{\mathrm{p}}^{\text {Sneddon }}\right)$ and Hertzian contact theory $\left(E_{\mathrm{p}}^{\text {Hertz }}\right)$ are expected to yield the same values.

Using Eq. (1) for pyramidal tips, the elastic modulus can be related to $H_{\mathrm{U}}$ by rearranging the Eq. (5) as

$$
H_{\mathrm{U}}=\frac{2 C \tan \alpha}{\pi\left(1-v_{\mathrm{p}}^{2}\right)} E_{\mathrm{p}}^{\text {Sneddon }},
$$

where $\frac{2 C \tan \alpha}{\pi\left(1-v_{\mathrm{p}}^{2}\right)}$ is a constant term and, consequently, $H_{\mathrm{U}}$ would be directly proportional to $E_{\mathrm{p}}^{\text {Sneddon }}$. The resulting trend would be in agreement with the experiments conducted with Berkovich tips as both $H_{\mathrm{U}}$ and $E_{\mathrm{p}}^{\text {Sneddon }}$ increases with decreasing $h$. Considering the influence of the above mentioned rotation gradients, a depth dependent hardness model corresponding to Eq. (7) has been suggested in [21]

$$
H_{\mathrm{U}}=H_{0}\left(1+\frac{c_{\ell}}{h}\right)
$$

where $H_{0}$ is the macroscopic hardness (considered as the lower limit of $H_{\mathrm{U}}$ ) and $c_{\ell}$ is a length scale parameter of a material which can be related to $\widehat{K}$ (see [21, 22] for details) as $c_{\ell}=\beta \widehat{K}$, with $\beta$ as a constant. $H_{0}=0.21 \mathrm{MPa}$ and $c_{\ell}=8.0 \mu \mathrm{m}$ are obtained from fitting Eq. (9) to data given in [13]. The 
hardness model Eq. (9) predicts that the hardness is proportional to $1 / h$ which is in agreement with increases in hardness with decreasing $h$ observed in the experiments mentioned in section 2.

The relations between tip geometries and rotation gradients in Fig. 2 and the hardness model of Eq. (9) are based on approximating assumptions. In order to corroborate these assumptions, numerical simulations based on the axisymmetric finite element [29] for the couple stress theory outlined above have been performed. To reduce computational expenses, axisymmetric simulations are preferred over 3 dimensional simulations. For metals, several authors [38, 39] have compared Berkovich indentation experimental results with numerical results under axisymmetric conditions. Similarly, numerical simulations with the rotation gradient theory are performed here under axisymmetric conditions (see Fig. 3 ), to compare them with experiments exhibiting length scale effects on elastomers. For the convenience of the reader, basic constitutive equations in axisymmetry are given in Table 1 and a very brief description of the numerical finite element approach is given below (please, see [29] for more details).

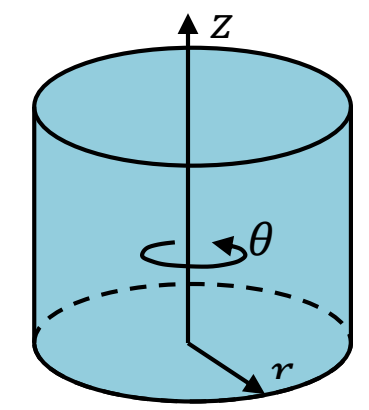

Fig. 3. Cylindrical coordinate system $(r, \theta, z)$

Table 1 Couple stress theory in cylindrical coordinates

- Infinitesimal rotation $\quad \omega_{\theta}=\frac{1}{2} \frac{\partial u_{z}}{\partial r}-\frac{1}{2} \frac{\partial u_{r}}{\partial z}$

- Strain vector components

$$
\boldsymbol{\varepsilon}=\left\{\begin{array}{llll}
\varepsilon_{r r} & \varepsilon_{z z} & \varepsilon_{\theta \theta} & \gamma_{r z}
\end{array}\right\}^{\mathrm{T}}
$$

where $\varepsilon_{r r}=\frac{\partial u_{r}}{\partial r}, \varepsilon_{z z}=\frac{\partial u_{z}}{\partial z}, \varepsilon_{\theta \theta}=\frac{u_{r}}{r}$ and $\gamma_{r z}=\left(\frac{\partial u_{r}}{\partial z}+\frac{\partial u_{z}}{\partial r}\right)$

- Rotation gradient components $\quad \chi=\left\{\begin{array}{ll}2 \chi_{r \theta} & 2 \chi_{z \theta}\end{array}\right\}^{\mathrm{T}}$

$$
\begin{aligned}
& \text { with } \chi_{r \theta}=\chi_{\theta r}=\frac{1}{4}\left[\left(\frac{\partial^{2} u_{r}}{\partial r \partial z}-\frac{\partial^{2} u_{z}}{\partial r \partial r}\right)-\frac{1}{r}\left(\frac{\partial u_{r}}{\partial z}-\frac{\partial u_{z}}{\partial r}\right)\right] \\
& \text { and } \chi_{z \theta}=\chi_{\theta z}=\frac{1}{4}\left(\frac{\partial^{2} u_{r}}{\partial z \partial z}-\frac{\partial^{2} u_{z}}{\partial r \partial z}\right)
\end{aligned}
$$

- Cauchy stress components

$$
\boldsymbol{\sigma}=\mathbf{C} \boldsymbol{\varepsilon}=\left\{\begin{array}{llll}
\sigma_{r r} & \sigma_{z z} & \sigma_{\theta \theta} & \sigma_{r z}
\end{array}\right\}^{\mathrm{T}}
$$

$$
\text { with } \mathbf{C}=\frac{E}{(1-2 v)(1+v)}\left[\begin{array}{cccc}
1-v & v & v & 0 \\
v & 1-v & v & 0 \\
v & v & 1-v & 0 \\
0 & 0 & 0 & \frac{1-2 v}{2}
\end{array}\right]
$$

- Couple stress components

$$
\mathbf{m}=\left\{2 m_{r \theta} \quad 2 m_{z \theta}\right\}^{\mathrm{T}}=2 \mu \ell^{2} \chi
$$

- Total potential energy

$$
\Pi\left(u_{r}, u_{z}\right)=\frac{1}{2} \int_{V} \boldsymbol{\varepsilon}^{\mathrm{T}} \mathbf{C} \boldsymbol{\varepsilon} d V+\mu \ell^{2} \int_{V} \boldsymbol{\chi}^{\mathrm{T}} \boldsymbol{\chi} d V-\int_{V} \mathbf{u}^{\mathrm{T}} \mathbf{f} d V-\int_{S} \mathbf{u}^{\mathrm{T}} \mathbf{t} d S-\int_{S} \omega q d S
$$


Under axisymmetric conditions, the displacement component $u_{\theta}$, displacement derivatives in $\theta$, and rotation components in $r$ and $z$ are 0, i.e. $u_{\theta}=0, \frac{\partial u_{r}}{\partial \theta}=\frac{\partial u_{z}}{\partial \theta}=0$ and $\omega_{r}=\omega_{z}=0$. Therefore, the only non-zero rotation component is given as $\omega=\omega_{\theta}$ in Eq. (10) of Table 1 and non-vanishing components of the strain tensor are contained in its vector representation in Eq. (11). The non-zero components of the rotation gradient tensor are shown in Eq. (12) and the Cauchy stress components are given in Eq. (13). The $\chi$ corresponding couple stress components can be found in Eq. (14). The total potential energy for axisymmetry is given in Eq. (15), where displacement components, body forces, tractions, and couple traction are denoted as $\mathbf{u}=\left\{\begin{array}{ll}u_{r} & u_{z}\end{array}\right\}^{\mathrm{T}}, \mathbf{f}=\left\{\begin{array}{ll}f_{r} & f_{z}\end{array}\right\}^{\mathrm{T}}, \mathbf{t}=\left\{\begin{array}{ll}t_{r} & t_{z}\end{array}\right\}^{\mathrm{T}}, q=q_{\theta}$, respectively.

To satisfy the $C^{1}$ continuity condition and to obtain a relatively simple numerical formulation, the approach in $[40,29]$ is adopted where a rotational degree of freedom $\phi=\phi_{\theta}$ (only non-zero rotation component) is introduced as an independent nodal variable. The rotation $\omega_{\theta}$ determined from $\mathbf{u}$ is replaced by $\phi_{\theta}$ for determining the rotation gradients. The difference between $\phi_{\theta}$ and $\omega_{\theta}$ is minimized by a penalty term (with $\alpha=\phi_{\theta}-\omega_{\theta}$ and a penalty parameter $P$ ) in the total potential energy [29] given for axisymmetric case as

$$
\Pi^{\mathrm{P}}(\mathbf{u}, \phi)=\frac{1}{2} \int_{V} \boldsymbol{\varepsilon}^{\mathrm{T}} \mathbf{C} \boldsymbol{\varepsilon} d V+\int_{V} \mu \ell^{2} \chi^{\phi^{\mathrm{T}}} \chi^{\phi} d V+\frac{P}{2} \int_{V} \alpha^{2} d V-\int_{V} \boldsymbol{u}^{\mathrm{T}} \mathbf{f} d V-\int_{S} \mathbf{u}^{\mathrm{T}} \mathbf{t} d S-\int_{S} \phi q d S
$$

whose corresponding finite element approach has been implemented into the ABAQUS user element interface (Dassault Systèmes Simulia Corp., Providence, RI, USA) and applied for the simulations below.

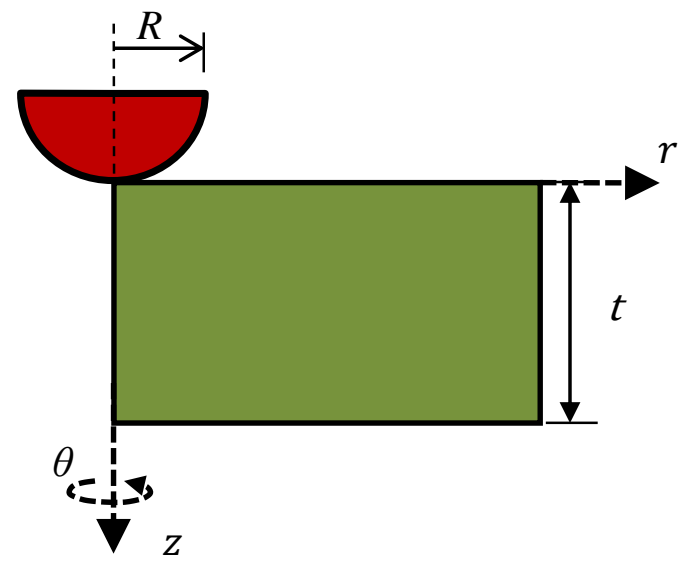

(a)

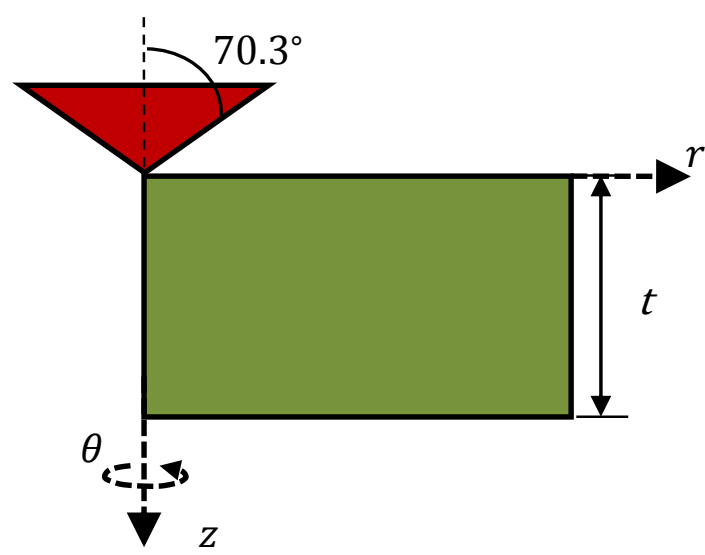

(b)

Fig. 4. Axisymmetric indentation with different indenter tip geometries: a) Spherical and b) Conical.

\section{Numerical results and comparison with experiments}

Axisymmetric indentation is considered where 2 different indenter tip geometries (spherical and conical) are applied on polymer cylinders of thickness $t$ (see Fig. 4). An elastic modulus of $E=2.2 \mathrm{MPa}$ is used for PDMS ( $E$ was obtained from the experiments in [13]), along with a Poisson's ratio of $v=$ 0.495 and a penalty parameter of $P=10^{5} \mu \ell^{2}$. The conical indenter tip is modeled as a perfectly rigid cone with a semi-vertical angle $\theta=70.3^{\circ}$, which results in the same contact area to depth ratio of a perfect Berkovich indenter. The spherical tips are also modeled to be perfectly rigid. Biased meshes (as shown in Fig. 5) are applied to discretize the polymer material, with well refined meshes underneath the indenter tip and coarser discretization farther away from it. The meshes are comprised of 10,000 eight- 
node quadrilateral elements. The stress/strain gradients are higher for the conical tip compared to the spherical tip. Correspondingly, the finite element meshes under the indenter of the conical tip are discretized finer. Overall finer meshes than the ones used did only negligible change the simulation results. Symmetric boundary conditions are applied at the axis of symmetry and fixed boundary conditions at the bottom of the sample. The boundary on the right edge is free. The radius and height of the specimen is equal to 90 and 70 times of the probing depth, respectively, for all numerical simulations to avoid any influence of the boundary at the right and the bottom of the specimen. The simulations are performed under displacement controlled conditions on a reference point specifying the movement of the tip surface. For a given indenter displacement, the corresponding load is determined by summing up the reaction forces (in $z$ direction) on the bottom nodes.

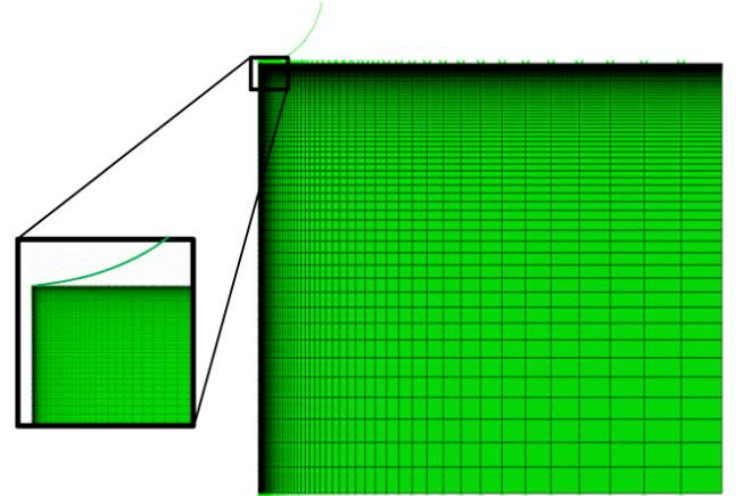

(a)

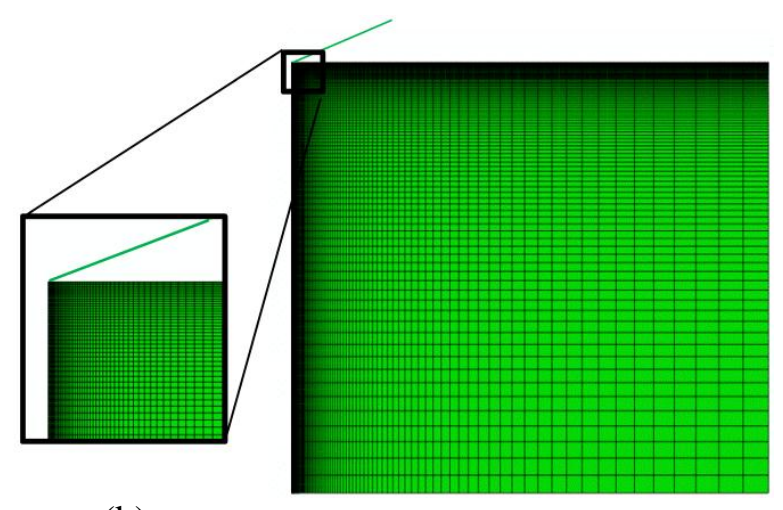

(b)

Fig. 5. Finite element mesh for the PDMS plate with both a) spherical and b) conical indenter tips.

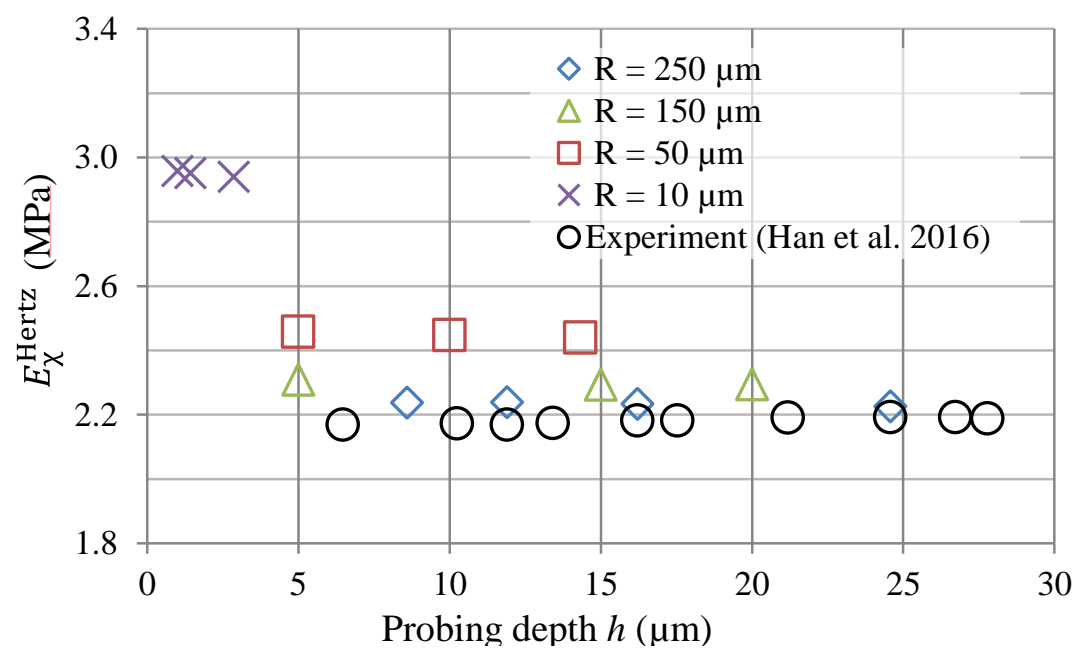

Fig. 6. Elastic modulus versus probing depth $h$ of PDMS using spherical tip with different radii.

\subsection{Spherical tip indentation testing}

Numerical simulations with different spherical indenter tip radii are performed on PDMS blocks for varying probing depth and the reaction force $F$ is determined for all the simulations. With the reaction force $F$ and corresponding indentation depth $h$ the elastic modulus is recalculated ( $E_{\chi}^{\mathrm{Hertz}}$ according to the Hertzian Eqs. (3) and (4)). $E_{\chi}^{\text {Hertz }}$ is depicted in Fig. 6 with varying $h$ for different indenter tip radii 
$R$. Experimental results for $R=250 \mu \mathrm{m}$ [13] are also redrawn in Fig. 6 and a good agreement between experiments and numerical simulations can be observed. With decreasing $R$ increases in the $E_{\chi}^{\text {Hertz }}$ can be found. However, for the same tip radius, $E_{\chi}^{\text {Hertz }}$ is almost constant with varying $h$, illustrating that the effect of the rotation gradients are negligible with respect to $h$. Similar trends have been observed in indentation experiments on epoxy [41] where the measured elastic modulus has been found to increase with the decreasing indenter tip radius but essentially remains independent of $h$ for a given tip radius.

$E_{\chi}^{\mathrm{Hertz}}$ is also plotted against the varying $R$ in Fig. 7. Therein, it can be seen that for $R>300 \mu \mathrm{m}$ the recalculated elastic modulus $E_{\chi}^{\text {Hertz }}$ is almost equal to the applied elastic modulus $E=2.2 \mathrm{MPa}$ obtained from experiments of [11]. However, $E_{\chi}^{\text {Hertz }}$ increases with decreasing $R$ indicating an increase in rotation gradients with decreasing $R$. It should be noted that in numerical simulations with spherical tips (illustrated in Fig. 7), the indentation depth is always about $29 \%$ of $R$.

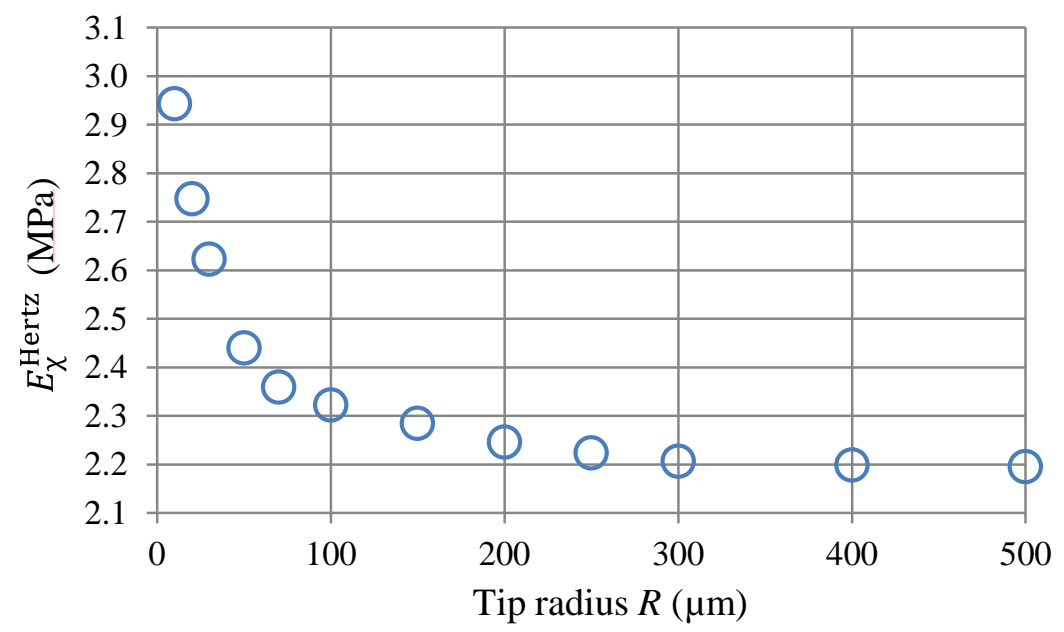

Fig. 7. $E_{\chi}^{\mathrm{Hertz}}$ against radius of spherical tip $R$ for a constant ratio of probing depth to $R(h / R=0.2867)$.
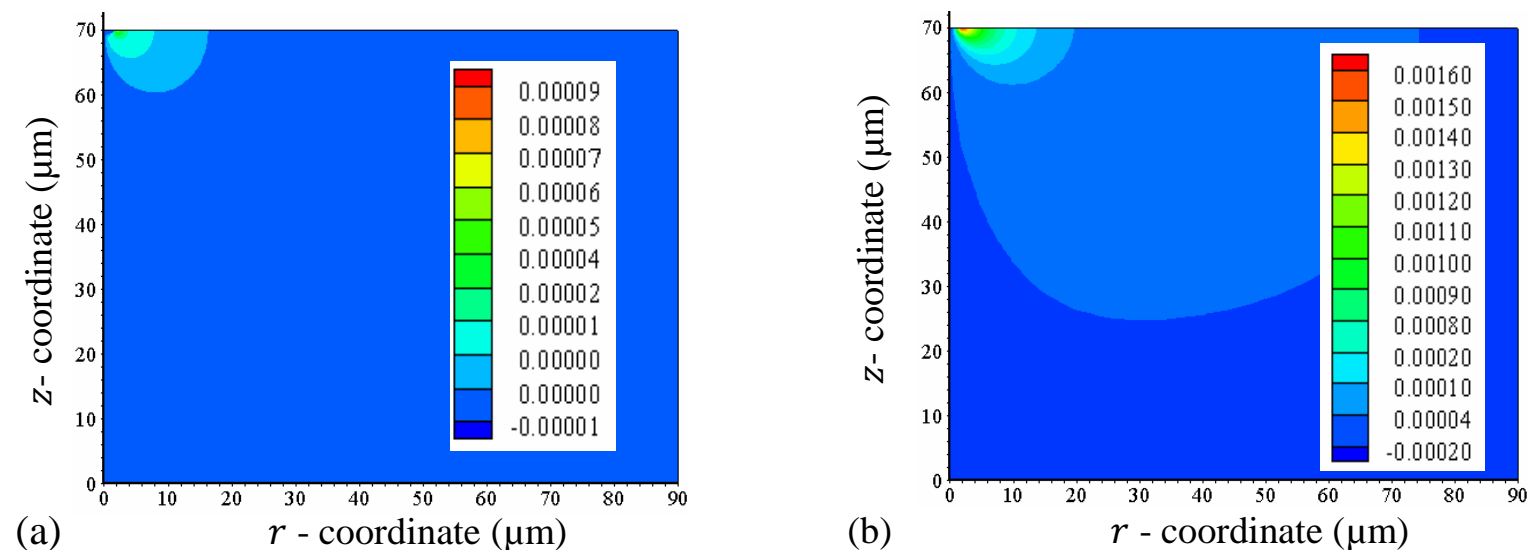

Fig. 8. Rotation gradient $\chi_{r \theta}$ for different spherical tip curvatures a) $R=500 \mu \mathrm{m}$ and b) $R=10 \mu \mathrm{m}$.

In Fig. 8, distribution of rotation gradient $\chi_{r \theta}$ is plotted for spherical tip radii (a) $R=500 \mu \mathrm{m}$ and (b) $R$ $=10 \mu \mathrm{m}$. Fig. 9 shows the distribution of the rotation gradient $\chi_{z \theta}$ under the spherical tip radii (a) $R=$ $500 \mu \mathrm{m}$ and (b) $R=10 \mu \mathrm{m}$. From both Figs. 8 and 9, it can be clearly observed that the rotation gradients under the spherical tip with $R=500 \mu \mathrm{m}$ is almost negligible compared to the ones with $R=10 \mu \mathrm{m}$, i.e. 
rotation gradients effect increases with decreasing $R$ of the spherical tip. The simulation results in Fig. 7 would also indicate that $R$ should be greater than $300 \mu \mathrm{m}$ to exclude the effect of rotation gradients in the determination of the elastic modulus of PDMS with the Hertzian theory.
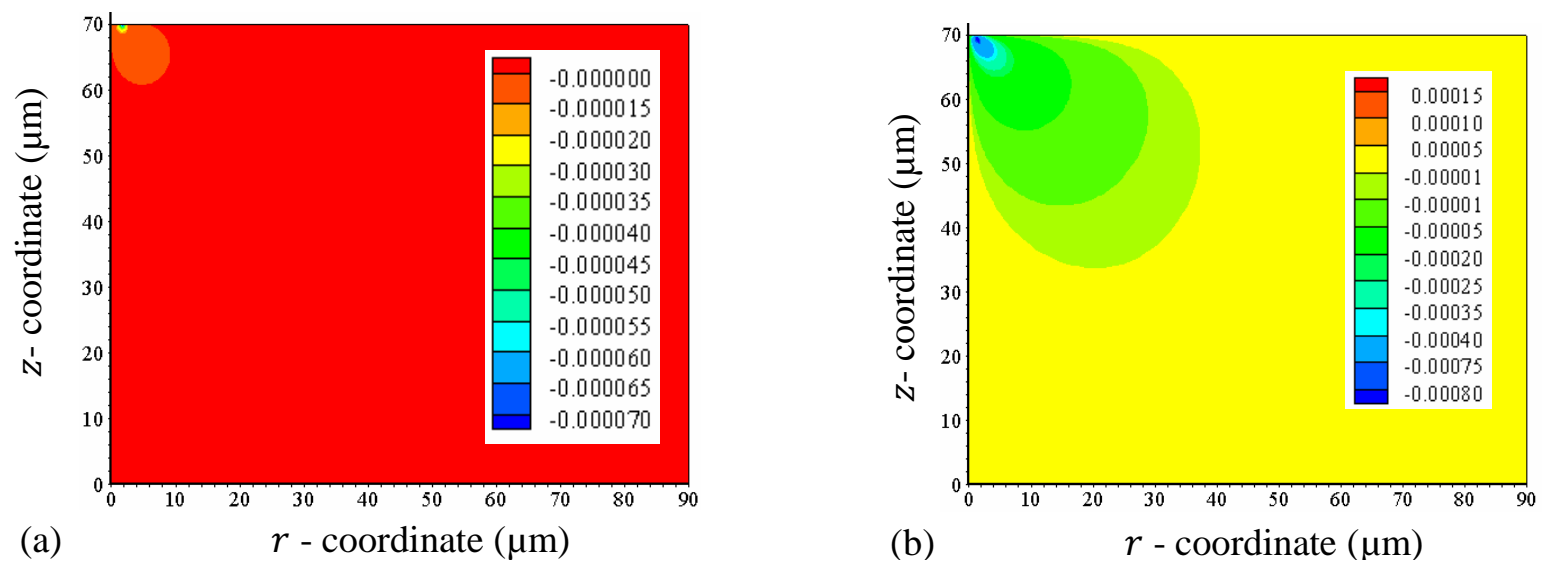

Fig. 9. Rotation gradient $\chi_{z \theta}$ for different spherical tip curvature a) $R=500 \mu \mathrm{m}$ and b) $R=10 \mu \mathrm{m}$.

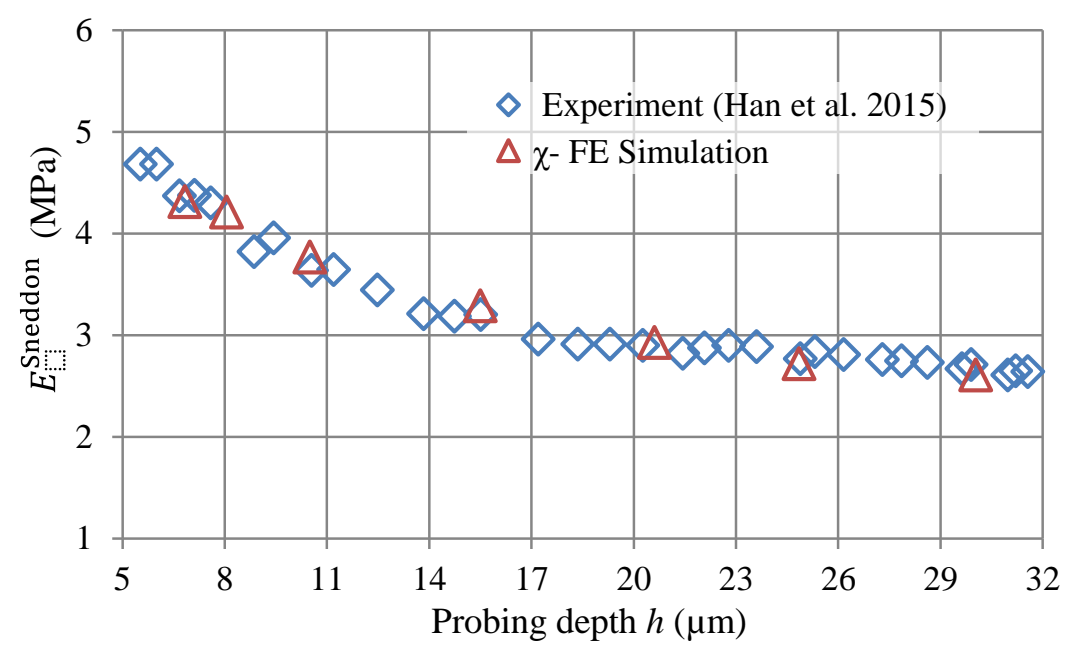

Fig. 10. $E_{\mathrm{p}}^{\text {Sneddon }}$ of PDMS versus $h$ determined with Sneddon's theory and conical tip.

\subsection{Conical tip indentation testing}

In order to illustrate the effect of higher order displacement gradients on the mechanical behavior of PDMS, numerical simulations with conical indenter tips are performed here for varying probing depths and the results are compared with the experimental results redrawn from [11]. Fig. 10 (where the elastic modulus determined via Sneddon's theory is plotted over $h$ ) and Fig. 11 (where the universal hardness is shown over $h$ ) clearly demonstrate the significance of the rotation gradient effects for $h$ ranging from 6 to $31 \mu \mathrm{m}$. The elastic modulus (determined with Sneddon's theory), $E_{\chi}^{\text {Sneddon }}$ is calculated from the reaction force obtained from numerical simulations using Eq. (5) and is plotted in Fig. 10. It can be seen in Fig. 10 that $E_{\chi}^{\text {Sneddon }}$ shows very good agreement with experimentally measured elastic modulus $E_{\mathrm{p}}^{\text {Sneddon }}$ redrawn from [11]. In Fig. 11, $H_{\mathrm{U}}$ determined with Eqs. (1) and (2) from the reaction force obtained from numerical simulations are shown over $h$. As classical, local continuum theories are length 
scale independent, it can be seen in Fig. 11 that without rotation gradients $H_{\mathrm{U}}$ remains constant for all the simulations and is identical to $H_{0}$ obtained from simulation where $\widehat{K}$ is set 0 . However, by including the rotation gradient effects $H_{\mathrm{U}}$ increases with decreasing $h$. Simulations applying the rotation gradient theory with $\widehat{K}=7.95 \mathrm{mN}$ result in good agreement between the obtained numerical values and the experimental data of $H_{\mathrm{U}}$ in PDMS for $h$ ranging between 6.8 to $31 \mu \mathrm{m}(\widehat{K}$ has been determined by fitting simulations to experimental data, i.e. $\widehat{K}$ has been varied in the finite element simulations till good agreement with experiments was found).

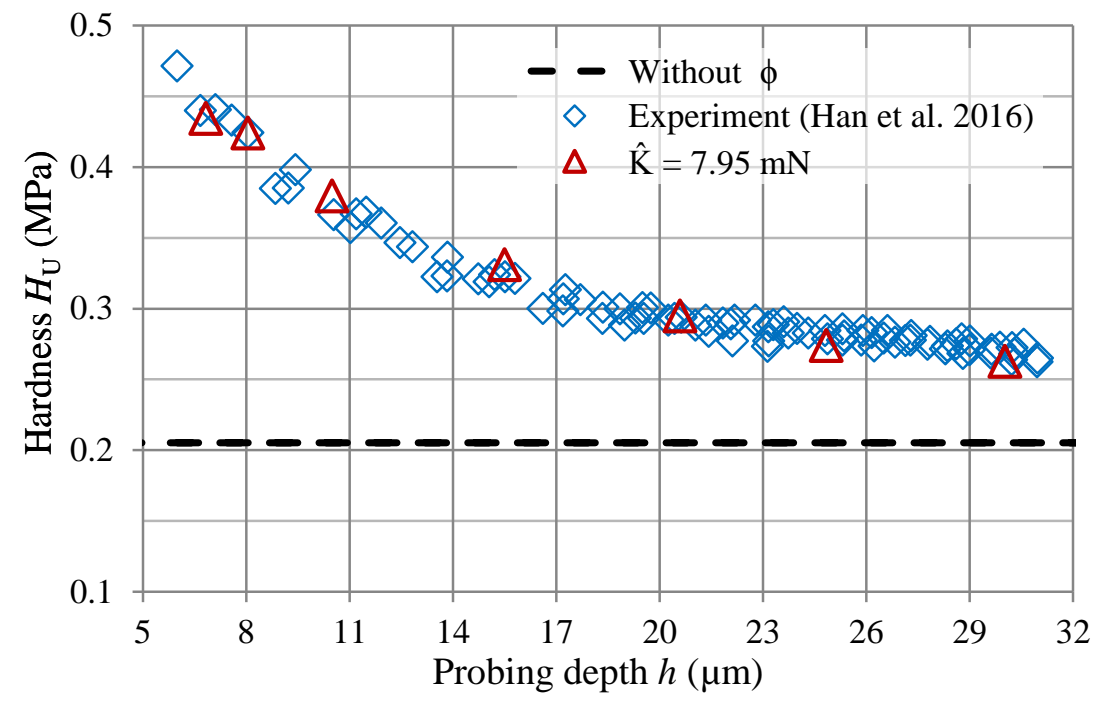

Fig. 11. Universal hardness $H_{\mathrm{U}}$ versus probing depth $h$ for PDMS with the conical indenter tip.

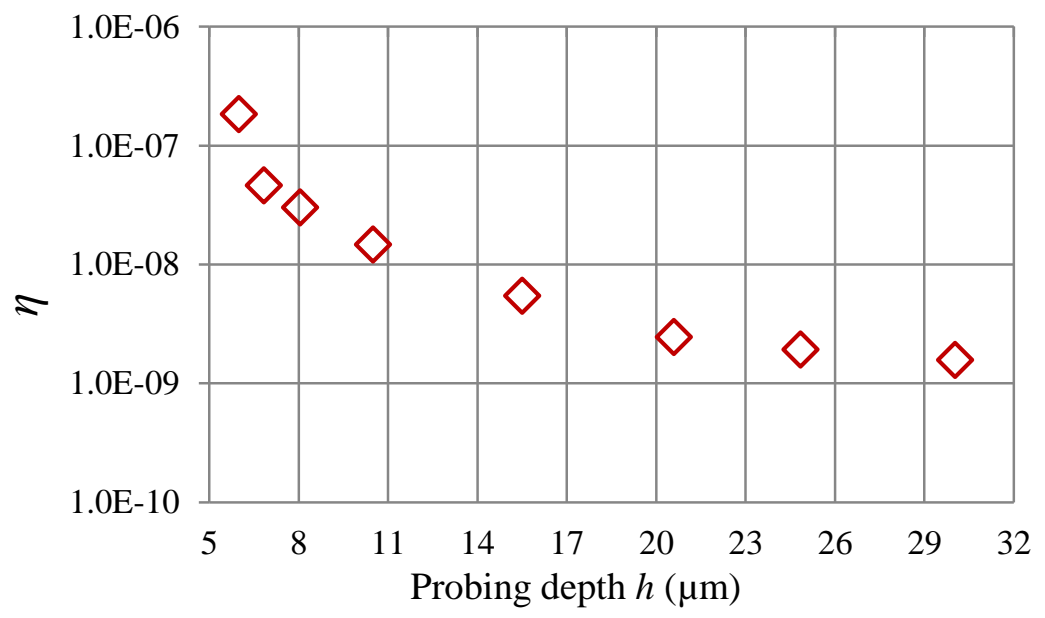

Fig. 12. Relative difference $\boldsymbol{\eta}$ versus probing depth $h$ for PDMS with the conical indenter tip.

Simulations for $h<6.8 \mu \mathrm{m}$ were affected by numerical problems, as with decreasing $h$ higher penalty parameters would have been necessary to enforce good agreement between nodal rotations $\phi_{\theta}$ and rotations determined from displacement fields $\omega_{\theta}$ in Eq. (16). Higher penalty parameters than the one used resulted, however, in ill-conditioning of the stiffness matrices [29], so that it could not be inverted in double precision. The relative difference in the rotations $\boldsymbol{\eta}=\left|\frac{\phi-\omega}{\omega}\right|$ is plotted in Fig. 12, where the 
increase in the relative difference with decreasing $h$ illustrates the inaccurate fulfillment of the constraint at small $h$. Similar to deviations in stresses observed for a plate with a hole problem [42], the numerically calculated hardness deviates from experiments at small $h$ due to poor satisfaction of the penalty constraint yielding a reduced stiffness in the simulation results. The corresponding simulation results for $h<6.8 \mu \mathrm{m}$ were therefore omitted in all Figures. The limitation of lower penalty parameters could be circumvented applying quadruple rather than double precision, as then the inversion of the stiffness matrix would still result in reliable results as discussed in [42], where linear, non-contact problems have been evaluated and compared in double as well as quadruple precision which is computationally very expensive and inapplicable to the ABAQUS user element interface.
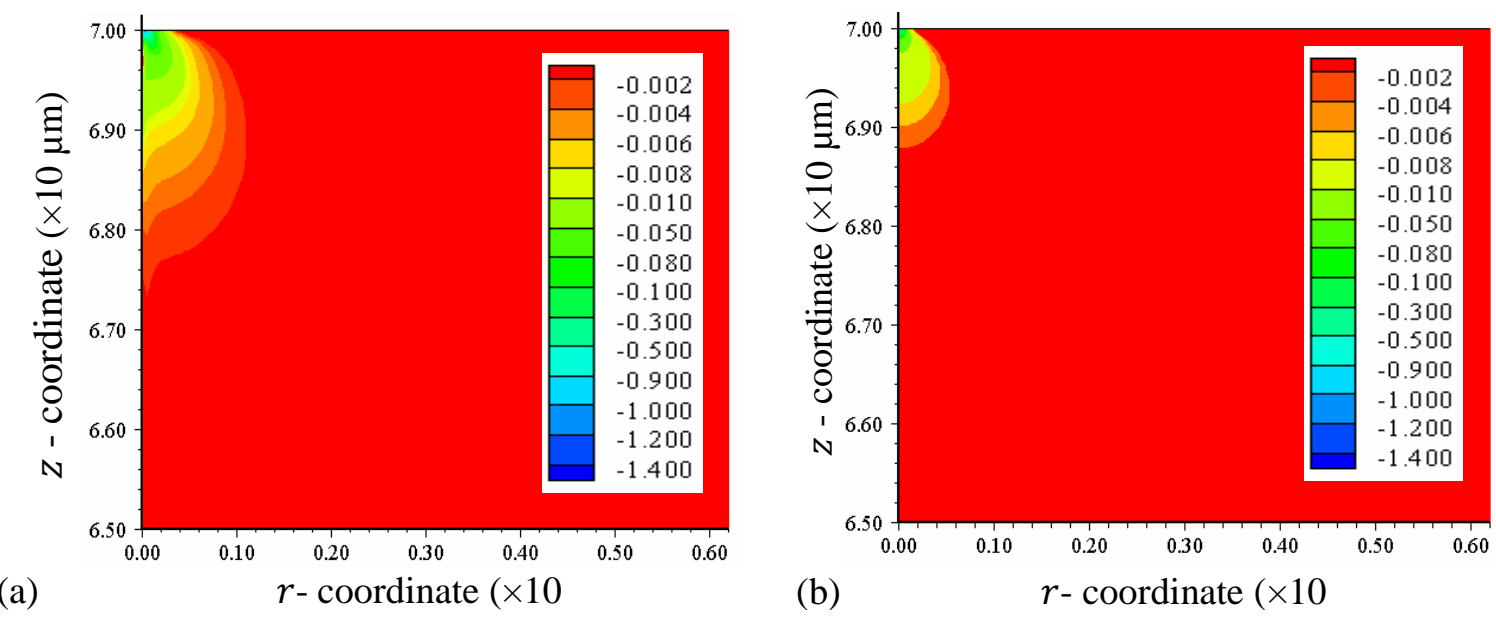

Fig. 13. Distribution of normal stress $\sigma_{z z}(\mathrm{MPa})$ in the vicinity of the conical indenter tip: (a) with rotation gradients and (b) without rotation gradients.
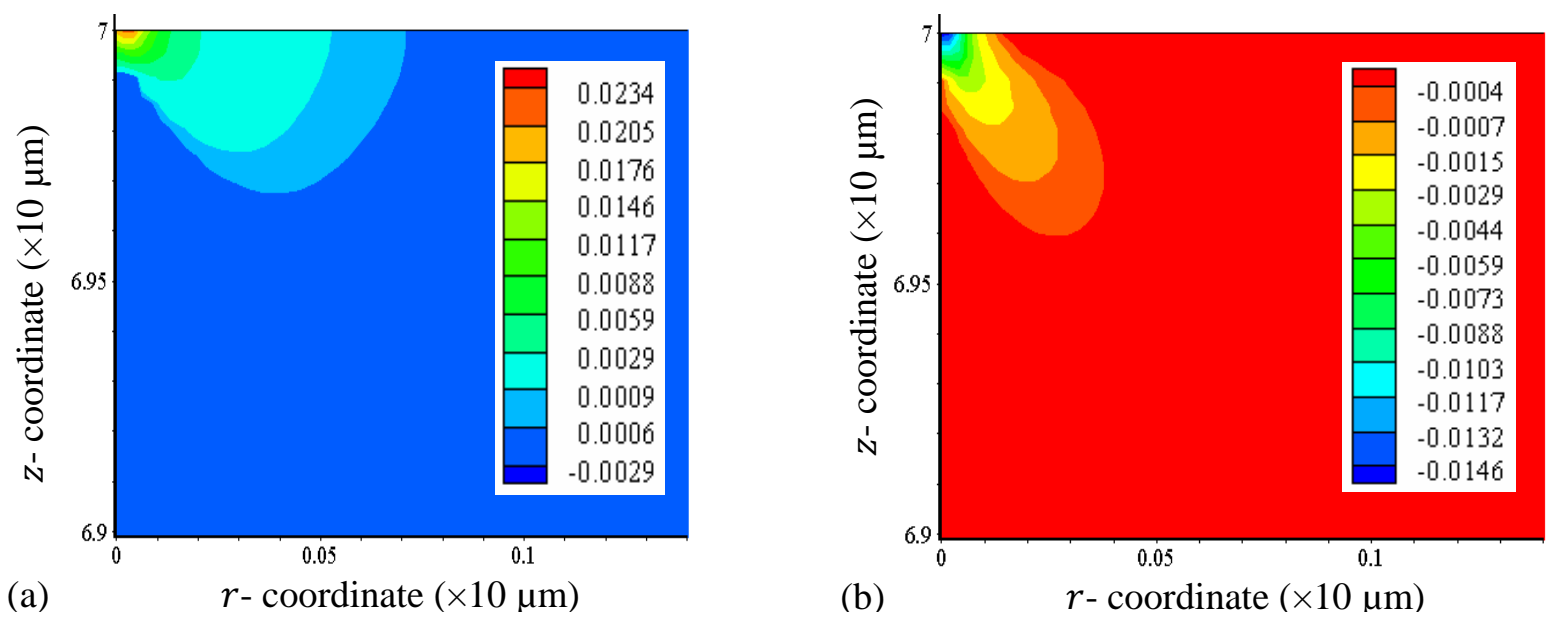

Fig. 14. Rotation gradients $(\mathrm{rad} / \mu \mathrm{m})$ in the vicinity of the conical indenter tip (a) $\chi_{r \theta}^{\phi}$ and (b) $\chi_{z \theta}^{\phi}$.

Fig. 13 illustrates the distribution of normal stress $\sigma_{z z}$ in the vicinity of the conical indenter tip at $h=$ $6.8 \mu \mathrm{m}$, with (a) and without (b) rotation gradient effects, respectively, where it can be seen that the maximum $\sigma_{z z}$ with rotation gradients are considerably higher, as compared to the ones obtained without rotation gradients. Fig. 14 shows the distribution of (a) $\chi_{r \theta}^{\phi}$ and (b) $\chi_{z \theta}^{\phi}$ under the conical indenter tip 
and it can be observed that significant rotation gradients are present at $h=6.8 \mu \mathrm{m}$. It can be concluded that $h$ dependent deformation of PDMS can be predicted by higher order displacement gradient models like the one presented here. Fig. 15 depicts $H_{\mathrm{U}}$ with respect to inverse of $h$ and it can be clearly observed that the theoretical hardness model defined in Eq. (9) shows very good agreement with hardness results obtained both experimentally and numerically.

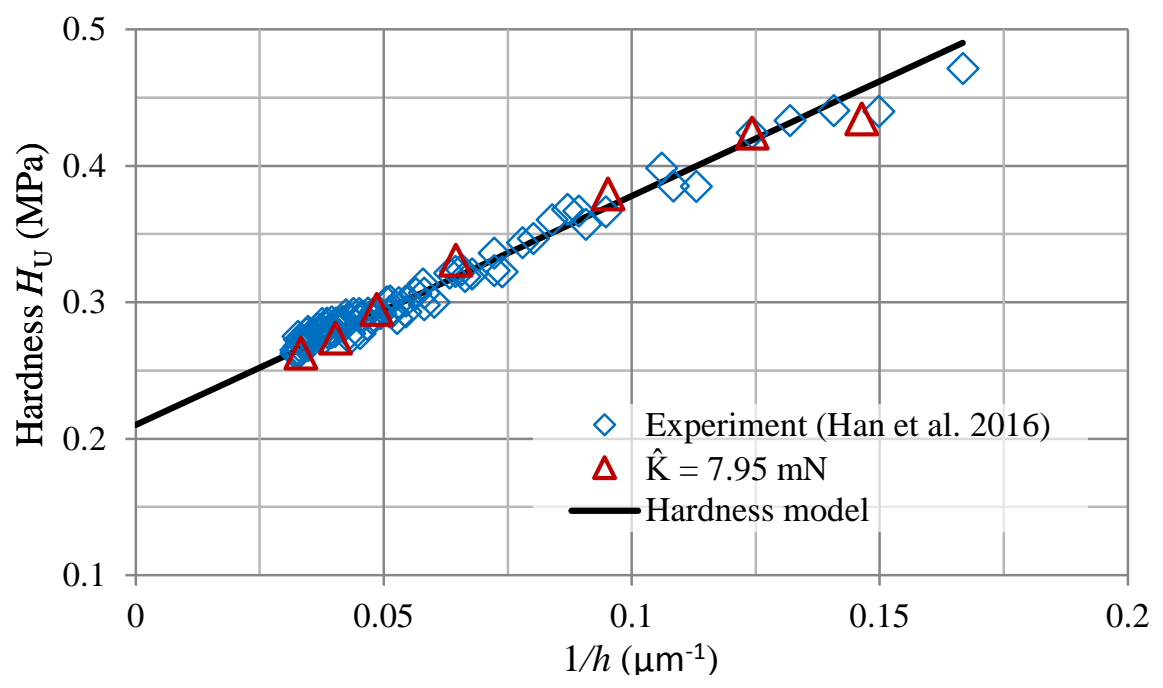

Fig. 15. Universal hardness $H_{\mathrm{U}}$ versus inverse of $h$ for PDMS with the conical indenter tip.

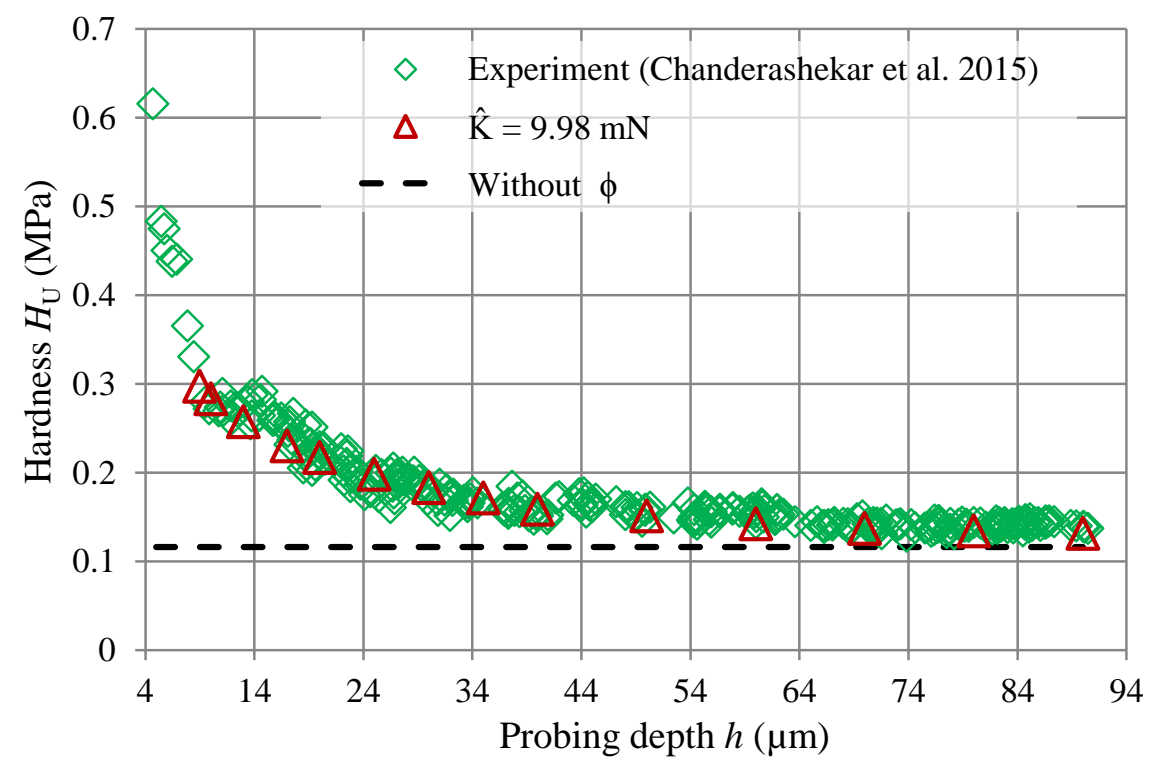

Fig. 16. Universal hardness $H_{\mathrm{U}}$ versus probing depth $h$ for natural rubber with the conical indenter tip.

\subsection{Conical tip indentation testing on natural rubber}

Similar to PDMS, size dependent behavior has been also experimentally observed in nanoindentation of natural rubber [14] at different probing depths. $H_{\mathrm{U}}$ for natural rubber at probing depth $h$ ranging from $4.7 \mu \mathrm{m}$ to $90 \mu \mathrm{m}$ obtained from [14] has been redrawn in Fig. 16 and it can be observed that $H_{\mathrm{U}}$ increases from $0.14 \mathrm{MPa}$ to $0.62 \mathrm{MPa}$ with decreasing depth from $90 \mu \mathrm{m}$ to $4.7 \mu \mathrm{m}$. 
Numerical simulations with a conical indenter tip are performed on natural rubber for varying $h$ (using $E=1.38 \mathrm{MPa}$ ), to compare simulation results with the experiments. Fig. 16 demonstrates the significance of rotation gradient effects for $h$ ranging from 9 to $90 \mu \mathrm{m}$. The incorporation of rotation gradients with $\widehat{K}$ $=9.98 \mathrm{mN}$ (determined by fitting to experimental data) results into a decent agreement between the obtained numerical and the experimental results of natural rubber for $h$ ranging between 9 to $90 \mu \mathrm{m}$. As for PDMS mentioned above, due to numerical limitations at shallow depths, numerical results below 9 $\mu \mathrm{m}$ are deviating from the experiments. In the case of natural rubber, as depicted in Fig. 17, the theoretical hardness model defined in Eq. (9) can also predict the depth dependent hardness with $H_{0}=$ $0.116 \mathrm{MPa}$ and $c_{\ell}=17.75 \mu \mathrm{m}($ or $\widehat{K}=9.98 \mathrm{mN})$.

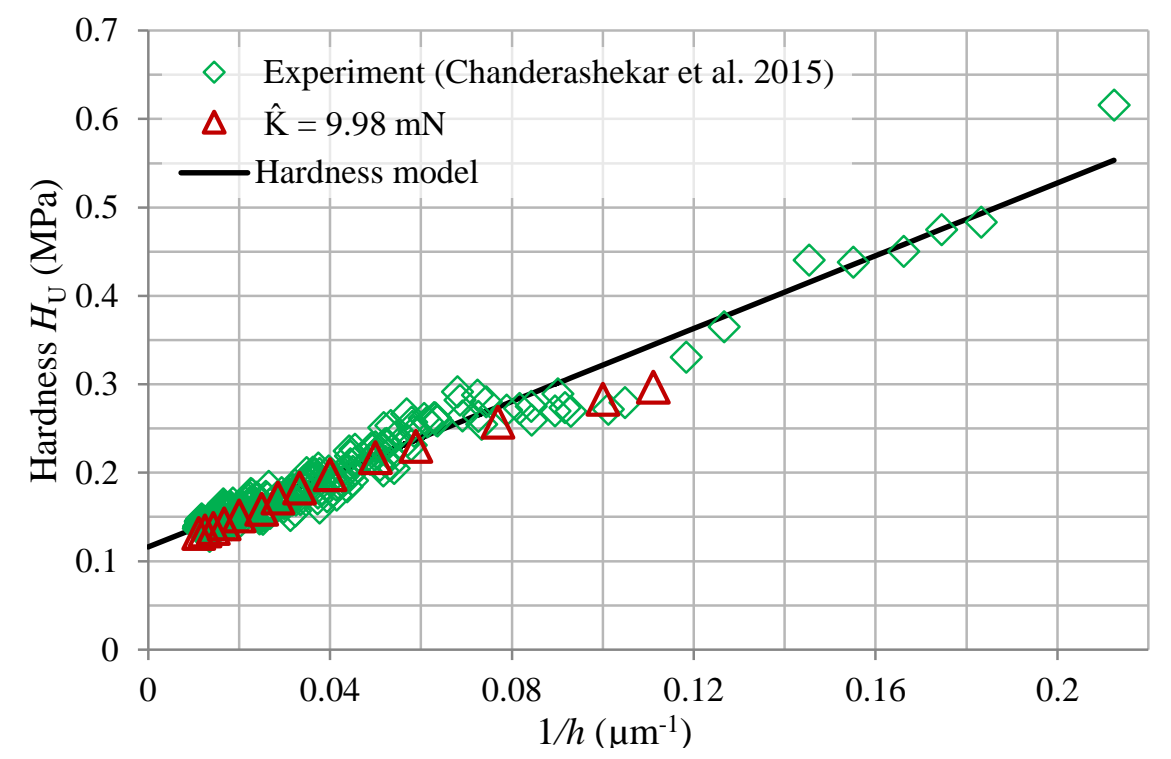

Fig. 17. Universal hardness $H_{\mathrm{U}}$ versus inverse of $h$ for natural rubber with the conical indenter tip.

\section{Conclusions}

Numerical simulations were performed to study the probing depth dependent behavior of PDMS applying spherical and conical indenter tips for a range of probing depths between 6 and $31 \mu \mathrm{m}$. Good agreement between the numerical simulations and experiments was observed for both the tips. For spherical indentations, it was numerically shown that the rotation gradients are unaffected by the probing depth which would corroborate the notion of the influence of higher gradients in the deformation mechanisms. The numerical results also showed the influence of the rotation gradients where stiffening was observed with decreasing radii of the spherical indenter tips. For indentations with the conical tip, numerical results for both PDMS and natural rubber are in good agreement with experimental results where a Berkovich tip was applied to probing depths as low as $6.8 \mu \mathrm{m}$ and $9 \mu \mathrm{m}$, respectively. Consequently, the rotation gradient rationale is applicable to explain the increase in hardness and elastic modulus determined by Sneddon's and Hertzian theories with decreasing probing depth.

\section{Acknowledgments}

Support of this work by the U.S. National Science Foundation, Grant CMMI 1102764, is highly appreciated. 


\section{References}

1. Ma Q, Clarke DR. J Mater Res 1995;10:853-863.

2. Nix WD, Gao H. J Mech Phy Sol 1998;46:411-425

3. Stelmashenko NA, Walls AG, Brown LM, Milman YV. Acta Metal Mater 1993;41:2855-2865.

4. Briscoe BJ, Fiori L, Pelillo E. J Phys D 1998;31:2395-2405.

5. Chong ACM, Lam DCC. J Mater Res 1999;14:4103-4110.

6. Lam DCC, Chong ACM. J Mater Res 1999;14:3784-3788.

7. Lam DCC, Chong ACM. Mater Sci Eng A 2000;281:156-161.

8. Tatiraju RVS, Han C-S, Nikolov S. Open Mech J 2008;2:89-92.

9. Lim YY, Chaudhri MM. Mech Mater 2006;38:1213.

10. Alisafaei F, Han CS, Lakhera N. Polym Test 2014;40:70-78.

11. Han CS, Sanei SHR, Alisafaei F. J Polym Eng 2016;36:103-111.

12. Alisafaei F, Han CS, Garg N. Int J Plast 2016;77, 30-53.

13. Alisafaei F, Han CS, Sanei SHR. Polym Test 2013;32:1220-1228.

14. Chandrasekhar G, Alisafaei F, Han CS. J Appl Polym Sci 2015;132:42683.

15. Han C-S. Mater Sci Eng A 2010;527:619-624.

16. Alisafaei F, Han CS. Adv Cond Mat Phys 2015;2015:391579 (20 pages).

17. Lam DCC, Yang F, Chong ACM, Wang J, Tong P. J Mech Phys Sol 2003;51:1477-1508.

18. McFarland AW, Colton JS. J Micromech Microeng 2005;15:1060-1067.

19. Tjernlund JA, Gamstedt EK, Gudmundson P. Int J Sol Struc 2006;43:7337-7357.

20. Shen L, Liu T, Lv P. Polym Test 2005;24:746-749.

21. Han CS, Nikolov S. J Mater Res 2007;22:1662-1672.

22. Nikolov S, Han CS, Raabe D. Int J Sol Struc 2007;44:1582-1592, Corrigendum: Int J Sol Struc 2007;44:7713.

23. Cosserat E, Cosserat F. Theorie des corps deformables, 1909, Hermann et Fils: Paris.

24. Mindlin RD, Tiersten HF. Arch Rat Mech Anal 1962;11:415-448.

25. Toupin RA. Arch Rat Mech Anal 1962;11:385+.

26. Koiter WT. Couple stresses in the theory of elasticity I and II. Proc K Ned Akad Wet B 1964;67:17-44.

27. Swaddiwudhipong S, Poha LH, Hua J, Liu ZS, Tho KK. Mater Sci Eng A 2005;404:179-187.

28. van Melick HGH, Bressers OFJT, den Toonder JMJ, Govaert LE, Meijer HEH. Polym Test 2003;44:2481-2491.

29. Garg N, Han CS. Arch Appl Mech 2015;85:587-600.

30. ISO 14577-1. Metallic materials - Instrumented indentation test for hardness and materials parameters - Part 1: test method. International Organization for Standardization 2002.

31. Oliver WC, Pharr GM. J Mater Res 1992;7:1564.

32. Hertz H. Journal für die Reine und Angewandte Mathematik 1882;29:156-171.

33. Sneddon IN. Int J Eng Sci 1965;3:47-57.

34. Ghosh S, Sundararaghavan V, Waas AM. Int. J. Sol. Struc. 2014;51:392-401.

35. Dai L, Sorkin V, Zhang YW. Polymer 2015; 68:11-16.

36. Gerberich WW, Tymiak NI, Grunlan JC, Horstemeyer MF, Baskes MI. ASME J Appl Mech 2002;69:433-442.

37. Yang F, Chong ACM, Lam DCC, Tong P. Int J Sol Struc 2002;39:2731-2743.

38. Celantano DJ, Guelorget B, Francois M, Cruchaga MA, Slimane A. Model Simul Mater Sci Eng 2012;20:045007.

39. Dao M, Chollacoop N, Van Vliet KJ, Venkatesh TA, Suresh S. Acta Mater 2001;49:3899-3918.

40. Garg N, Han CS. Comput Mech 2013;52,709-720.

41. Chandrasekhar G, Han CS. Length scale effects and the dependence of elastic moduli measurements on the spherical indenter tip radii in epoxy, 2016, submitted.

42. Garg N. Finite Element Modeling of the Length Scale Dependent Deformation in Polymers, 2015, Ph.D. dissertation, Dept Mech Eng, University of Wyoming, Adviser: C.-S. Han. 

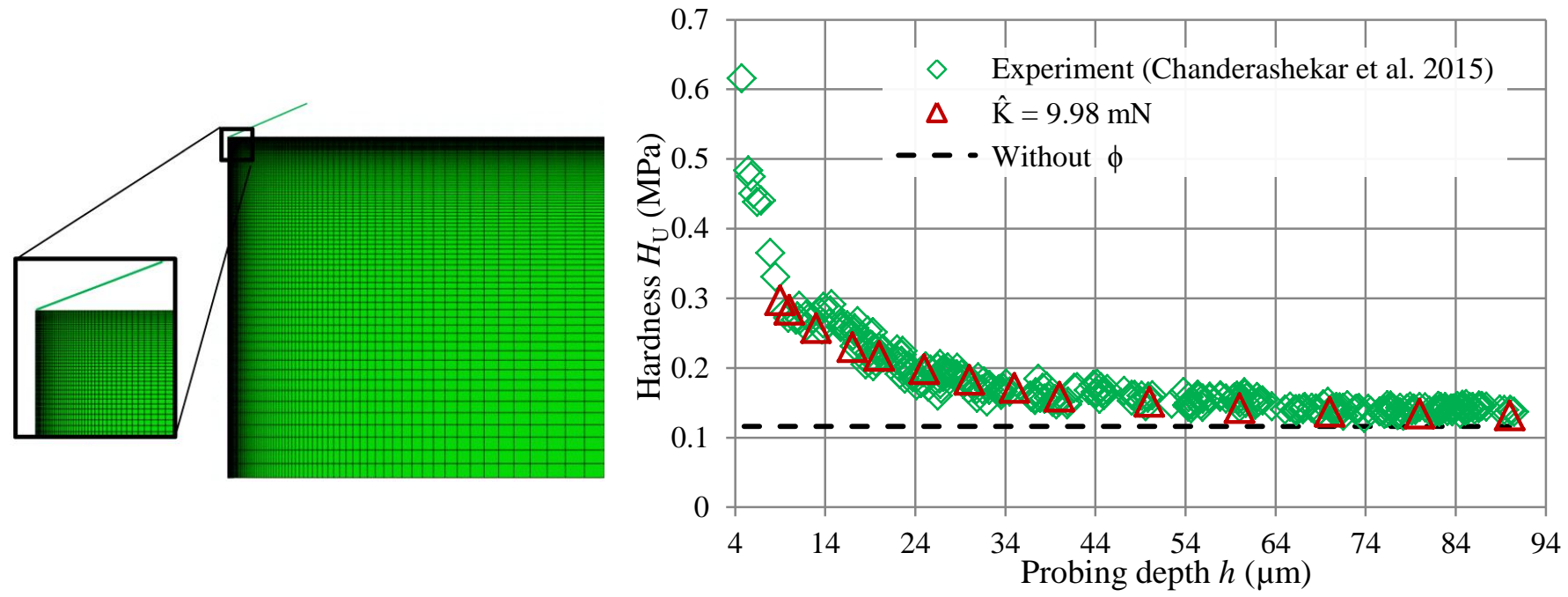